\title{
Densidade populacional de raposa-do-campo Lycalopex vetulus (Carnivora, Canidae) em áreas de pastagem e campo sujo, Campinápolis, Mato Grosso, Brasil
}

\author{
Ednaldo C. Rocha ${ }^{1}$, Elias Silva ${ }^{1}$, Renato N. Feio ${ }^{2}$, Sebastião V. Martins ${ }^{1} \&$ Gisele Lessa $^{2}$
}

1. Departamento de Engenharia Florestal e Pós-graduação em Ciência Florestal da Universidade Federal de Viçosa, DEF/UFV, 36570-000 Viçosa, MG. (ednaldorocha@yahoo.com.br)

2. Departamento de Biologia Animal da Universidade Federal de Viçosa, DBA/UFV, 36570-000 Viçosa, MG.

\begin{abstract}
Population density of the hoary fox, Lycalopex vetulus (Carnivora, Canidae), in pasture and campo sujo areas in Campinapolis, Mato Grosso, Brazil. In face of the growing disturbance of the Cerrado biome, due to the expansion of the agricultural borders of Central Brazil, it becomes important to evaluate the adaptation capacity of animal species to the anthropized environment. Thus, this work was developed aiming to estimate and compare the population density of hoary fox Lycalopex vetulus (Lund, 1842) in two areas of Central Brazil with distinct disturbance degrees, pasture and campo sujo, a vegetation type of Cerrado. For this, during the period of August to November 2005, nocturnal sampling were done along linear transects, totaling $129.8 \mathrm{~km}$ in the campo sujo and $62.08 \mathrm{~km}$ in the pasture. A total of 23 sightings of hoary fox was obtained in campo sujo and 52 in the pasture. Estimations of population density were made using the Distance 5.0 software, which chose the half normal + hermite adjust as the model with best fit. Population density was higher in the pasture $\left(D=4.28\right.$ individuals $\left./ \mathrm{km}^{2} ; \mathrm{IC}=2.69-6.82\right)$ than in the campo sujo $\left(\mathrm{D}=1.21\right.$ individuals $/ \mathrm{km}^{2}$; IC $=0.73-2.01$ ), what is likely related, mainly, to higher availability of food and reduction of potential predators. As its diet is composed basically of termites, especially from the genera Syntermes and Cornitermes, the hoary fox find in the pasturage area an alimentary base abundant and stable. Moreover, the environmental simplification, due to the pasture implantation, end up reducing or to local extinction potential predators of the hoary fox, such as Chrysocyon brachyurus (Illiger, 1815), supporting the increase of population density of this specie in this kind of environment. Results indicate that the Cerrado endemic hoary fox is able to survive, and even proliferate, in highly modified areas of the Cerrado.
\end{abstract}

KEYWORDS. Campo sujo, pasture, population density, hoary fox, Lycalopex vetulus.

RESUMO. Diante da crescente descaracterização do Bioma Cerrado em função da expansão da fronteira agropecuária na região central do Brasil, torna-se importante avaliar a capacidade de adaptação das espécies ao ambiente antropizado. Neste sentido, este trabalho foi desenvolvido com o objetivo de estimar e comparar a densidade populacional da raposa-do-campo Lycalopex vetulus (Lund, 1842) em duas áreas com diferentes graus de alteração, pastagem e campo sujo, em Campinápolis, Mato Grosso. Para tanto, no período entre agosto a novembro de 2005, foram efetuados censos noturnos ao longo de transectos lineares, totalizando percursos de $129,8 \mathrm{~km}$ na área de campo sujo e $62,08 \mathrm{~km}$ na área de pastagem. Estimativas de densidade populacional foram geradas utilizando o programa Distance 5.0, sendo que o modelo e ajuste mais adequados aos dados foram half-normal + hermite. Foram obtidas 23 e 52 detecções de raposas-docampo nas áreas de campo sujo e pastagem, respectivamente. A densidade populacional de raposa-do-campo na área de pastagem $(\mathrm{D}=4,28$ indivíduos $/ \mathrm{km}^{2} ; \mathrm{IC}=2,69-6,82$ ) foi maior que na área de campo sujo ( $\mathrm{D}=1,21$ indivíduos $/ \mathrm{km}^{2} ; \mathrm{IC}=0,73-2,01$ ), fato que deve estar relacionado, principalmente, com a disponibilidade de alimento e redução de potenciais predadores. Por apresentar uma dieta composta principalmente de cupins, especialmente os dos gêneros Syntermes e Cornitermes, a raposa-do-campo encontra na área de pastagem uma base alimentar abundante e estável. Além disto, a simplificação ambiental, em função da implantação de pastagens acaba por reduzir, ou até mesmo eliminar, animais que são potenciais predadores de raposas-do-campo, como Chrysocyon brachyurus (Illiger, 1815), favorecendo o aumento da densidade populacional da espécie neste tipo de ambiente. Por fim, características adaptativas apresentadas pela raposa-docampo têm permitido que esta espécie sobreviva, inclusive apresentando elevada densidade populacional, em áreas de pastagem utilizadas para a criação de gado, em Campinápolis, Mato Grosso, onde a vegetação original era Cerrado.

PALAVRAS-CHAVE. Campo sujo, pastagem, densidade populacional, raposa-do-campo, Lycalopex vetulus.

O Cerrado está localizado basicamente no Planalto Central do Brasil e constitui-se na segunda maior formação vegetal brasileira, ocupando cerca de 2 milhões de $\mathrm{km}^{2}$ (aproximadamente $23 \%$ do território nacional) e apresentando fisionomias vegetais que englobam formações florestais, savânicas e campestres (RIBEIRO \& Walter, 1998). Nos últimos anos, esse bioma tem enfrentado rápida redução de sua cobertura vegetal original devido à expansão da fronteira agropecuária na região central do Brasil. Cerca de metade da cobertura original do Cerrado já foi transformada em pastagens plantadas, culturas anuais e outros tipos de uso. As pastagens plantadas com gramíneas de origem africana cobrem atualmente cerca de $500.000 \mathrm{~km}^{2}$ (KLINK \& Machado, 2005). Diante do quadro da crescente descaracterização dos ecossistemas originais do Cerrado, torna-se importante avaliar a capacidade de adaptação das espécies ao ambiente antropizado, visando traçar estratégias de conservação.

Mamíferos com ocorrência no Cerrado totalizam cerca de 195 espécies, sendo que 18 destas são endêmicas (MMA, 1999). Quanto aos canídeos, quatro espécies habitam esse bioma, lobo-guará (Chrysocyon brachyurus (Illiger, 1815)), cahorro-do-mato-vinagre (Speothos venaticus (Lund, 1842)), cachorro-do-mato (Cerdocyon thous (Linnaeus, 1766)) e raposa-do-campo (Lycalopex vetulus (Lund, 1842)). Esta última espécie é a menor delas e tem ocorrência confirmada apenas para o Brasil, onde vive em áreas de formações vegetais abertas do Planalto Central e áreas periféricas de transição (DALPONTE \& COURTENAY, 2004). 
A raposa-do-campo (L. vetulus) não está listada como ameaçada de extinção no Brasil (MMA, 2003) e na avaliação do seu status pela IUCN (The World Conservation Union) consta como "dados insuficientes", ou seja, não há informações adequadas para se fazer uma avaliação sobre seu risco de extinção. Nos últimos anos, essa espécie tem sido alvo de alguns estudos englobando aspectos ecológicos, biológicos e conservacionistas (DAlPonte, 1995; 1997; DALPONTE \& Lima, 1999; JuAREZ \& Marinho-Filho, 2002; JÁcomo et al., 2004; CourTENAY et al., 2006). No entanto, estimativas de densidade populacional ainda não haviam sido realizadas.

Assim, o objetivo deste estudo foi estimar e comparar a densidade populacional de L. vetulus em duas áreas do bioma Cerrado, em Campinápolis, Mato Grosso, com diferentes graus de alteração (pastagem e campo sujo).

\section{MATERIAL E MÉTODOS}

Áreas de estudo. A coleta de dados foi realizada em duas áreas, campo sujo e pastagem, localizadas no município de Campinápolis, leste do estado de Mato Grosso, centro-oeste do Brasil (Fig.1). A distância entre elas é de cerca de $35 \mathrm{~km}$, em linha reta.

As áreas amostradas estão inseridas no bioma Cerrado e apresentam clima do tipo Aw (tropical estacional), segundo a classificação de Köppen, sendo caracterizado por duas estações bem definidas, uma seca (de abril a setembro) e uma chuvosa (de outubro a março) (NIMER, 1989). A precipitação pluviométrica média anual é de aproximadamente $1.500 \mathrm{~mm}$, com médias de temperaturas em torno de $25,5^{\circ} \mathrm{C}$, sendo junho e julho os meses mais frios e setembro o mais quente (PINTO JUNIOR \& Rossete, 2005).

O relevo da região é caracterizado como plano a ondulado, apresentando altitudes entre 250 e $300 \mathrm{~m}$ e com ocorrências de solos litólicos, cambissolo transicional com deposição de blocos de arenito e Latossolo Vermelho-Amarelo (RADAMBRASIL, 1981).

Área de campo sujo. Campo sujo (Fig. 2) é um tipo fisionômico exclusivamente herbáceo-arbustivo, com arbustos e subarbustos esparsos cujas plantas, muitas vezes, são constituídas por indivíduos menos desenvolvidos das espécies arbóreas do cerrado sensu stricto (RIBEIRO \& WALTER, 1998). O local amostrado ( $\left.14^{\circ} 17^{\prime} \mathrm{S}, 52^{\circ} 56^{\prime} \mathrm{W}\right)$ tem uma área estimada de 2.500 ha, pertencentes à Reserva Indígena "Parabubure" (224.447 ha). Essa área está localizada a cerca de $38 \mathrm{~km}$ da cidade de Campinápolis e, segundo informações de antigos moradores da região, em 1967 já se encontrava habitada pelos índios Xavante. Atualmente, a aldeia possui aproximadamente 130 pessoas e encontra-se a $3 \mathrm{~km}$ do início do sítio amostrado.

Embora a vegetação natural da área de estudo ainda não tenha sido removida, é freqüente a ocorrência de distúrbios ocasionados por queimadas que a atingem total ou parcialmente. $\mathrm{O}$ fogo, que acaba se espalhando por extensas áreas, é utilizado principalmente durante rituais de caça dos índios e na limpeza e renovação de pastagem.

Área de pastagem. Trata-se de uma área de 1.300 ha, que engloba 12 propriedades privadas de pequeno e médio porte $\left(14^{\circ} 33^{\prime} \mathrm{S}\right.$ e $\left.52^{\circ} 46^{\prime} \mathrm{W}\right)$, cuja paisagem é dominada por pastagens de gado coberta com espécies de capins africanos, especialmente Brachiaria sp. (Poaceae). Em sua porção inicial, a área localiza-se a cerca de $2 \mathrm{~km}$ da cidade de Campinápolis (Fig. 3). Atualmente, o município apresenta uma população estimada em 13.000 habitantes, sendo a pecuária a base da economia local.

Segundo informações de antigos moradores locais, a cobertura vegetal original da área amostrada era cerrado sensu stricto, com a presença de várzeas nos locais mais baixos. Em 1978, a vegetação nativa foi removida para a introdução de lavoura de arroz e, dois anos depois, a

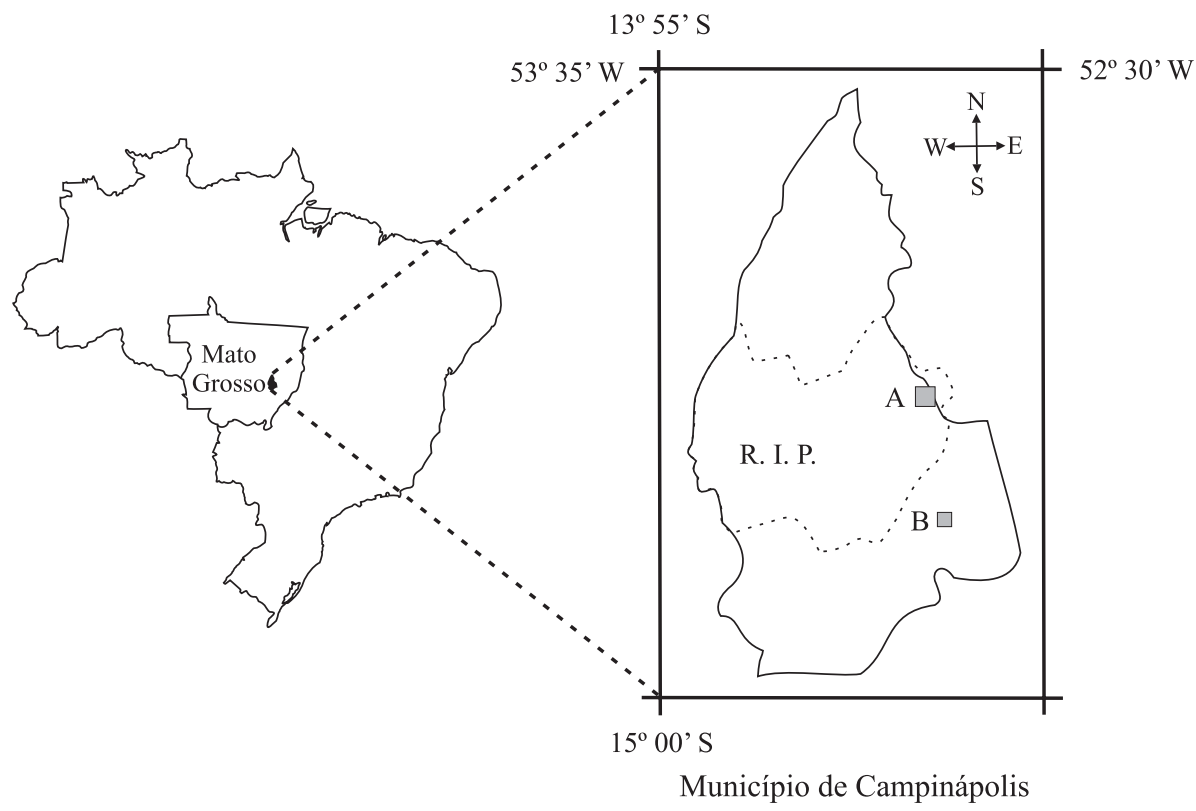

Fig. 1. Localização das áreas de estudo, campo sujo (A) e pastagem (B), no município de Campinápolis, Mato Grosso. Adaptado do IBGE (2006). (R. I. P., Reserva Indígena "Parabubure"). 


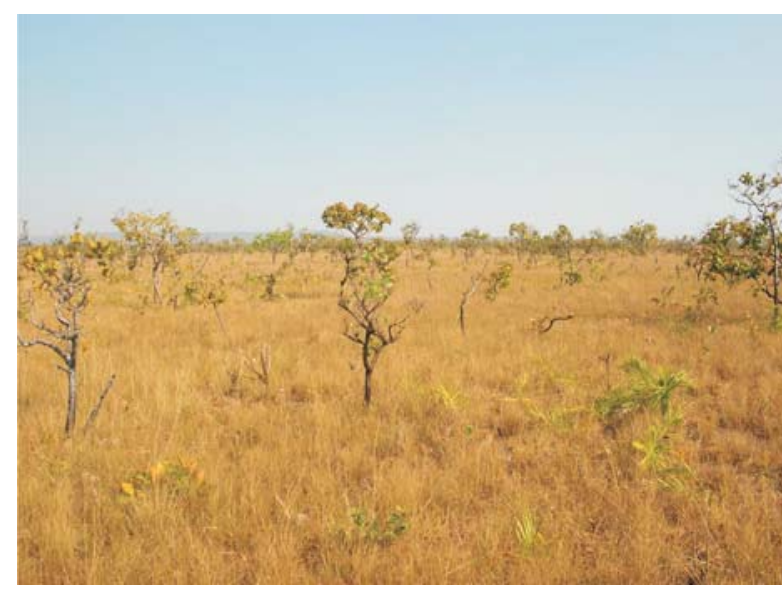

Fig. 2. Área de campo sujo na Reserva Indígena "Parabubure", Campinápolis, Mato Grosso.

orizicultura foi substituída por pastagens para a criação de gado, como permanece até a atualidade.

Coleta dos dados. A coleta de dados ocorreu no período entre 12 de agosto a 24 de novembro de 2005 , baseando-se nos princípios da metodologia Distance de estimadores de densidade, propostos por BUCKLAND et al. (1993) е Thомаs et al. (2002), os quais têm sido amplamente utilizados em estudos sobre mamíferos no Brasil (ChiARELlo, 2000; TomÁs et al., 2001; HAUGAASEN $\&$ Peres, 2005).

Transectos lineares foram percorridos durante caminhadas em dupla (numa velocidade média de $2,7 \mathrm{~km} / \mathrm{h}$ ), para a realização de censos noturnos, utilizando lanternas de longo alcance, alimentadas por quatro pilhas alcalinas grandes, para avistar os animais, binóculo para auxiliar na identificação e GPS para orientação linear ao longo do transecto e para medir a distância total de cada transecto.

Os transectos foram estabelecidos de forma a cobrir a máxima porção da área de estudo, mantendo uma distância de 500m entre eles, no caso de transectos paralelos amostrados na mesma noite. Além disto, sempre que o ambiente comportava, o comprimento de cada transecto foi superior a $1 \mathrm{~km}$.

Os censos, totalizando 35 noites de amostragem, ocorreram principalmente durante as primeiras horas da noite (19:00-00:00), coincidindo com o horário em que $L$. vetulus se encontra em franca atividade (JUAREZ \& Marinho-Filho, 2002; JÁcomo et al., 2004). Priorizou-se as noites mais escuras para realização dos censos, na medida em que nestas a eficiência da lanterna era aumentada e a detecção dos animais facilitada.

A cada detecção, foi medida a distância perpendicular entre o animal observado e o transecto, através da contagem de passos do observador que, posteriormente, foram convertidos em metros (Tomás et al., 2001). O fator de conversão consistiu no comprimento médio da passada, previamente estabelecido para cada um dos observadores.

De acordo com peculiaridades de cada área, a amostragem ocorreu conforme descrito a seguir:

Área de campo sujo. A coleta de dados foi efetuada no período entre 12 de agosto a 27 de setembro de 2005 , quando foram amostrados $129,8 \mathrm{~km}$ de transectos (comprimento médio=1,88km; desvio padrão=0,99km;

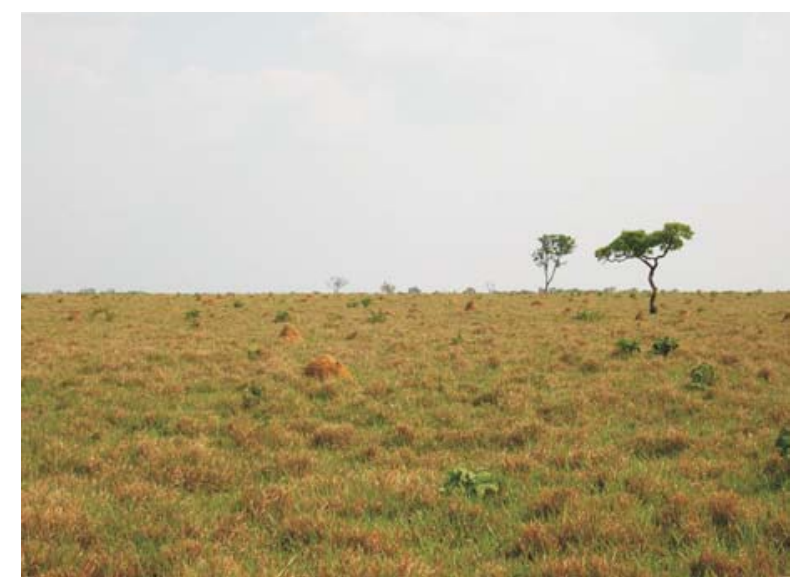

Fig. 3. Área de pastagem (Brachiaria sp.) próxima à Campinápolis, Mato Grosso.

variação: 0,67 a 4,70km, $\mathrm{n}=69$ transectos), durante 22 noites de trabalho. Os transectos lineares foram conduzidos ao longo de partes relativamente retilíneas de estradas e trilhas antigas e, em menor quantidade, percursos lineares em meio à vegetação, onde não havia acessos.

Área de pastagem. A coleta de dados foi efetuada no período entre 06 de outubro a 24 de novembro de 2005, quando foram amostrados $62,08 \mathrm{~km}$ de transectos (comprimento médio $=1,63 \mathrm{~km}$; desvio padrão $=0,65 \mathrm{~km}$; variação: 0,84 a $3,72 \mathrm{~km}, \mathrm{n}=38$ transectos), durante 13 noites de trabalho. Transectos lineares foram conduzidos em meio à pastagem, não utilizando estradas existentes na área por serem bastante movimentadas, podendo afugentar os animais. O primeiro transecto amostrado em cada noite foi escolhido arbitrariamente e os demais eram traçados paralelamente a este, mantendo 500m de distância entre eles.

Análise dos dados. Para estimar a densidade populacional de L. vetulus, a análise estatística foi conduzida utilizando o programa Distance 5.0, disponibilizado gratuitamente no sítio: http:// ww.ruwpa.st-and.ac.uk/distance (THOMAs et al., 2005). O fundamento desse método é a busca de um modelo, ou uma Função de Detecção, que melhor espelhe o comportamento das distâncias observadas. Depois, utiliza-se essa função para estimar a proporção de indivíduos que não foram detectados durante o censo. A partir daí pode-se obter uma estimativa de densidade da população de interesse (BuCKLAND et al., 1993; ТномAS et al., 2002; CULLEN \& RUdRAN, 2003).

A metodologia Distance para a amostragem em transectos lineares assume quatro premissas, em ordem decrescente de importância: (1) todos os animais posicionados diretamente na linha do transecto devem ser detectados; (2) todos os animais são detectados na sua posição inicial, antes de qualquer movimento em resposta ao observador; (3) as distâncias perpendiculares são medidas corretamente e (4) as detecções são eventos independentes (BUCKLAND et al., 1993; ThOMAs et al., 2002; Cullen \& RudRan, 2003).

A partir dos dados obtidos em campo, simulações foram efetuadas com todos os modelos disponíveis no programa Distance 5.0, com dados truncados e não truncados. A escolha do modelo que melhor se ajustou 
aos dados levou em consideração dois fatores: mínimo AIC (Akaike's Information Criterion) (AкAIKE, 1974; Buckland et al., 1993; Thomas et al., 2002; Cullen \& RUDRAN, 2003; JATHANNA et al., 2003) e menor coeficiente de variação (CV), de forma a eleger um único modelo para as áreas de estudo e permitir comparações entre elas.

Observando os histogramas de distribuição das distâncias perpendiculares (Figs. 4, 5) e os resultados das simulações, optou-se por não truncar os dados, considerando todos os dados de distâncias perpendiculares, o que acarretaria um aumento do intervalo de confiança e do coeficiente de variação. $\mathrm{O}$ modelo e ajuste mais adequados aos dados foram halfnormal + hermite.

Adicionalmente, foi aplicado o teste qui-quadrado $\left(\chi^{2}\right)$ para verificar se houve diferença significativa entre os valores observados em campo e os esperados para as classes de distâncias perpendiculares no intuito de detectar possível ocorrência de viés amostral.

\section{RESULTADOS E DISCUSSÃO}

$\mathrm{Na}$ área de campo sujo foram obtidas 23 detecções de L. vetulus. Muito embora o número mínimo de detecções independentes recomendável seja 40, tamanhos amostrais menores, de até 20 detecções, também podem gerar estimativas robustas, dependendo da distribuição dos dados (BucKland et al., 1993; Peres, 1999; Cullen \& RudRan, 2003), como ocorreu com os dados coletados na área de campo sujo.

A densidade de L. vetulus estimada para a área de campo sujo foi de 1,21 (intervalo de confiança IC $=0,73$ 2,01 ) indivíduos $/ \mathrm{km}^{2}$ (Tab. I). Desta forma, a área amostrada (aproximadamente 2.500ha) apresenta uma população estimada em $30(\mathrm{IC}=18-50)$ indivíduos. No entanto, como essa área está inserida numa matriz ambiental maior (a Reserva Indígena "Parabubure"), estima-se que a população de raposas-do-campo na região seja maior.

$\mathrm{Na}$ área de pastagem foram obtidas 52 detecções de $L$. vetulus, onde a densidade estimada foi de 4,28 ( $\mathrm{IC}=2,69-6,82$ ) indivíduos $/ \mathrm{km}^{2}$ (Tab. I). Assim, a área

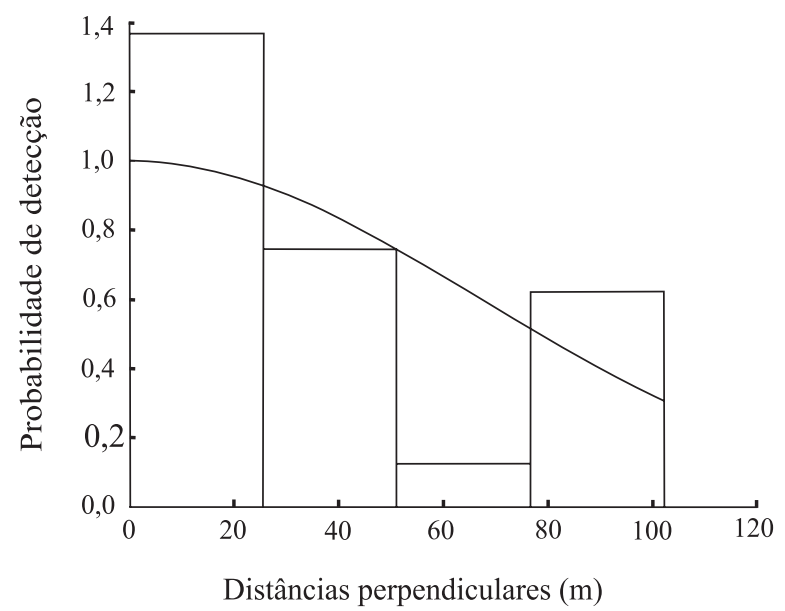

Fig. 4. Probabilidade de detecção e distâncias perpendiculares de registros de Lycalopex vetulus (Lund, 1842) na área de campo sujo, em Campinápolis, Mato Grosso. amostrada (com cerca de 1.300 ha) apresenta uma população estimada em 56 (IC=35-89) indivíduos.

Embora não haja trabalhos publicados que permitam comparar a densidade populacional de L. vetulus, os resultados deste estudo revelam uma elevada densidade populacional dessa espécie em áreas de pastagem de Campinápolis. Conforme DalPonte \& CourTenay (2004), a raposa-do-campo parece se adaptar bem à áreas de pastagem de gado ricas em cupins e besouros.

Observando o histograma de distribuição das distâncias perpendiculares para os dados da área de pastagem nota-se que houve elevada concentração de detecções próximas ao transecto (Fig. 4), indicando possível ocorrência de viés amostral. Entretanto, o teste qui-quadrado mostrou que não houve diferença significativa $\left(\chi^{2}=2,54 ; p=0,28\right)$ entre o número observado e esperado para os intervalos de distâncias perpendiculares. Por outro lado, na área de campo sujo ocorreram relativamente poucas observações próximas ao transecto (Fig. 5), o que pode ser um indício de fuga dos animais antes de serem detectados pelo observador (Jathanna et al., 2003). No entanto, essa possível violação de uma das premissas do método não foi confirmada, já que não houve diferença significativa, pelo teste qui-quadrado $\left(\chi^{2}=5,53 ; \mathrm{p}=0,06\right)$, entre o número observado e esperado para os intervalos de distâncias perpendiculares.

Comparação entre as áreas de campo sujo e de pastagem. Ao contrário do esperado para a maioria das espécies de mamíferos, a densidade populacional de $L$. vetulus na área de pastagem, provavelmente um ambiente com maior potencial alimentício, foi maior que na área de campo sujo (Tab. I).

A dieta de L. vetulus é típica de um forrageador onívoro, sendo constituída principalmente de cupins ceifadores. Além de cupins e outros artrópodes, frutas, pequenos mamíferos, pássaros e répteis também são utilizados por essa espécie como fonte alimentícia (DAlPonte, 1997; Dalponte \& Lima, 1999; Juarez \& MARINHO-FILHO, 2002; JÁcOMO et al., 2004). Besouros são consumidos em grandes quantidades, quando sazonalmente abundantes (DALPONTE \& COURTENAY, 2004).

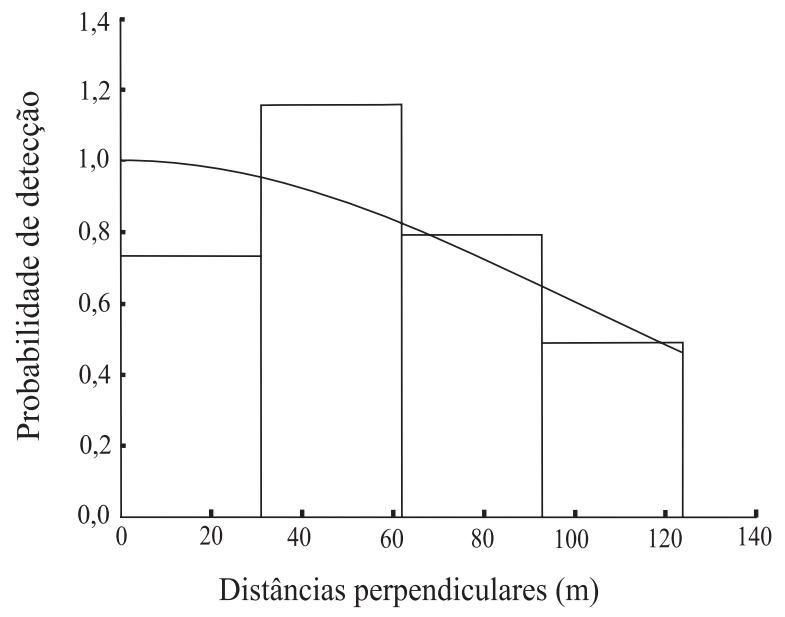

Fig. 5. Probabilidade de detecção e distâncias perpendiculares de registros de Lycalopex vetulus (Lund, 1842) na área de pastagem em Campinápolis, Mato Grosso. 
Tabela I. Estimativa de densidade populacional de Lycalopex vetulus (Lund, 1842) em duas áreas em Campinápolis (N, número de detecções; CV, coeficiente de variação; IC, intervalo de confiança, com probabilidade de $95 \%$ ).

\begin{tabular}{lccccc} 
Área amostrada & $\mathrm{N}$ & $\begin{array}{c}\text { Densidade } \\
\text { (indivíduos } / \mathrm{km}^{2} \text { ) }\end{array}$ & $\begin{array}{c}\text { Erro } \\
\text { padrão }\end{array}$ & $\mathrm{CV}(\%)$ & $\mathrm{IC}$ \\
\hline Campo sujo & 23 & 1,21 & 0,31 & 25,8 & $0,73-2,01$ \\
Pastagem & 52 & 4,28 & 1,01 & 23,7 & $2,69-6,82$ \\
\hline
\end{tabular}

A bula auditiva relativamente grande pode ser uma adaptação especial de L. vetulus que fornece maior acuidade auditiva, facilitando a localização das concentrações de cupins na superfície do solo, na medida em que essa espécie, aparentemente, utiliza uma combinação de som e odor emitidos pelos térmites para localizá-los (Dalponte, 1997). Dentes carniceiros pequenos e molares proporcionalmente maiores que em qualquer outra espécie de canídeo vivente (LANGGUTH, 1975; Stains, 1975) também parecem ser adaptações ao tipo de dieta predominantemente insetívora da raposado-campo (DALPONTE \& COURTENAY, 2004), tendo em vista que as superfícies de oclusão dos molares mais largas possibilitam romper, com maior facilidade, a carapaça dos besouros.

Devido à adaptação a uma dieta composta principalmente de cupins, $L$. vetulus encontra na área de pastagem uma base alimentar abundante e estável (Constantino, 2002; CzEPAK et al., 2003), fato que pode estar contribuindo para a manutenção de uma elevada densidade populacional da espécie nesse ambiente. Ademais, o esterco de bovinos acumulado nas pastagens funciona como um atrativo a outros insetos, como besouros, que também são consumidos em grandes quantidades pela raposa-do-campo (DALPONTE \& Courtenay, 2004).

Por outro lado, a simplificação ambiental, em função da implantação de pastagens, acaba por reduzir, ou até mesmo eliminar, animais que são potenciais predadores de L. vetulus, como C. brachyurus (JÁcomo et al., 2004), favorecendo o aumento da densidade populacional da espécie. Além disto, outro fator a ser considerado é a presença de cães domésticos, que podem atuar como predadores de raposas-do-campo (DALPONTE \& Courtenay, 2004). De fato, haviam cães domésticos tanto na área de campo sujo como na área de pastagem. Na propriedade onde haviam cães de caça não foi obtido nenhum registro de raposa-do-campo, embora o ambiente mostrava-se adequado à ocorrência dessa espécie.

A redução na quantidade de potenciais predadores aliada à alta disponibilidade de alimento podem estar contribuindo para a manutenção de uma densidade populacional de $L$. vetulus mais elevada nas áreas de pastagem que em áreas de campo sujo, em Campinápolis (Tab. I).

As queimadas que ocorrem periodicamente na área de campo sujo parecem não causar um efeito negativo direto sobre $L$. vetulus, na medida em que elas são capazes de refugiar-se em tocas. Além disto, como as queimadas são freqüentes, há pouco acúmulo de massa vegetal combustível, de forma que o fogo não alcança grandes proporções. Por outro lado, no período pós-fogo, certamente há redução na quantidade de alimento e abrigo disponíveis, causando efeitos adversos sobre a população de raposas-do-campo.

Deve ser considerado, também, que o período em que este estudo foi realizado coincidiu com a fase reprodutiva de L. vetulus, cujos nascimentos ocorrem uma vez ao ano, durante os meses de julho e agosto (Dalponte \& Courtenay, 2004; Courtenay et al., 2006). Desta forma, estimativas efetuadas após o período de dispersão dos indivíduos jovens poderão gerar densidades populacionais um pouco diferentes das encontradas no presente estudo. Além disto, não é descartada a possibilidade de que o estabelecimento dos transectos de forma diferenciada nas áreas amostradas possa ter influenciando os resultados.

Face ao exposto, percebe-se que L. vetulus possui características adaptativas que a permite sobreviver, inclusive com elevadas densidades populacionais, onde a cobertura da vegetação original de Cerrado (cerrado sensu stricto e campo sujo) foi substituída por pastagem para a criação de gado em Campinápolis, Mato Grosso.

Recomenda-se estudos sobre densidade populacional de L. vetulus por tempo mais prolongado, visando detectar flutuações populacionais ao longo do tempo e entre as estações. Além disto, é desejável que estimativas populacionais sejam conduzidas também em outras áreas, incluindo Unidades de Conservação e ambientes em regeneração.

Agradecimentos. Somos gratos ao Sr. Claudemiro Gomes da Silva e à Sra. Maria Lúcia Alves da Silva pela acolhida em sua residência e pelo inestimável auxílio durante toda a coleta de dados na área de campo sujo; ao amigo Julio Cesar Dalponte pelas valiosas sugestões e pela cessão de material indispensável, utilizado durante a coleta de dados; ao Paulo de Marco Jr. pela revisão do manuscrito; à CAPES pela bolsa de estudos concedida ao primeiro autor; aos proprietários da área de pastagem por autorizarem a realização da coleta de dados em seus imóveis rurais e aos revisores anônimos pelas valiosas sugestões.

\section{REFERÊNCIAS BIBLIOGRÁFICAS}

AKaike, H. 1974. A new look at the statistical model identification. IEEE Transactions on Automatic Control AC 19:716-723. Buckland, S. T.; Anderson, D. R.; Burnham, K. P. \& LaAke, J. L. 1993. Distance Sampling. Estimating Abundance of Biological Populations. London, Chapman \& Hall. 446p. Chiarello, A. G. 2000. Density and population size of mammals in remnants of brasilian Atlantic Forest. Conservation Biology 14(6): 1649-1657.

Constantino, R. 2002. The pest termites of South America: taxonomy, distribution and status. Journal of Applied Entomology 126:355-365.

Courtenay, O.; Macdonald, D. W.; Gillingham, S.; Almeida, G. \& Dias, R. 2006. First observations on South America's largely insectivorous canid: the hoary fox (Pseudalopex vetulus). Journal of Zoology 268(1):45-54.

Cullen, L. \& Rudran, R. 2003. Transectos lineares na estimativa de densidade de mamíferos e aves de médio e grande porte. In: Cullen, L.; Rudran, R. \& Valladares-Pádua, C. orgs. Métodos de estudos em Biologia da Conservação e Manejo da Vida Silvestre. Curitiba, UFPR. p.169-179.

Czepak, C.; Araúso, E. A.; Fernandes, P. M. 2003. Ocorrência de espécies de cupins de montículo em pastagens no estado de Goiás. Pesquisa Agropecuária Tropical 33(1):35-38.

Dalponte, J. C. 1995. The hoary fox in Brazil. Canid News 3:23-24.

1997. Diet of the hoary fox, Lycalopex vetulus, in Mato Grosso, Central Brazil. Mammalia 61(4):537-546. 
Dalponte, J. C. \& Lima, E. S. 1999. Disponibilidade de frutos e a dieta de Lycalopex vetulus (Carnivora - Canidae) em um cerrado em Mato Grosso, Brasil. Revista Brasileira de Botânica 22(2):325-332.

Dalponte, J. C. \& Courtenay, O. 2004. Hoary fox Pseudalopex vetulus (Lund, 1842). In: Sillero-Zubiri, C.; Hoffmann, M. \& MacDonald, D. W. eds. Canids: Foxes, Wolves, Jackals and Dogs. Status Survey and Conservation Action Plan. IUCN/SSC Canid Specialist Group, Gland, Switzerland and Cambridge, UK. p.72-76.

Haugaasen, T. \& Peres, C. A. 2005. Mammal assemblage structure in Amazonian flooded and unflooded forests. Journal of Tropical Ecology 21:133-145.

IBGE (Instituto Brasileiro de Geografia e Estatística) 2006. Mapas Interativos. Disponível em: <http://mapas.ibge.gov.br/>. Acesso em: 15.04.2006.

Jácomo, A. T. A.; Silveira, L. \& Diniz-Filho, J. A. F. 2004. Niche separation between the maned wolf (Chrysocyon brachyurus), the crab-eating fox (Dusicyon thous) and the hoary fox (Dusicyon vetulus) in central Brazil. Journal of Zoology 262:99-106.

Jathanna, D.; Karanth, K. U. \& Johnsingh, A. J. T. 2003. Estimation of large herbivore densities in the tropical forests of southern India using distance sampling. Journal of Zoology 261:285-290.

Juarez, K. M. \& Marinho-Filho, J. 2002. Diet, habitat use, and home ranges of sympatric canids in Central Brazil. Journal of Mammalogy 83(4):925-933.

Klink, C. A. \& Machado, R. B. 2005. A conservação do Cerrado brasileiro. Megadiversidade 1(1):147-155

LangGuth, A. 1975. Ecology and evolution in the South American canids. In: Fox, M. W. ed. The wild canids: their systematics behavioral ecology and evolution. New York, Van Nostrand Reinhold Company, p.192-206.

MMA - Ministério do MeIo Ambiente. 1999. Ações prioritárias para a conservação da biodiversidade do Cerrado Pantanal. Brasília, MMA/FUNATURA/Conservation International/ Fundação Biodiversitas, Universidade de Brasília. 26p. 2003. Espécies da Fauna Brasileira Ameaçadas de Extinção.
Instrução Normativa $\mathbf{n}^{\circ} \mathbf{3}$, Diário Oficial da União $\mathrm{n}^{\circ} 101$, de 28 de maio de 2003, Seção 1. p.88-97.

Nimer, E. 1989. Climatologia do Brasil. Rio de Janeiro, IBGE. $422 \mathrm{p}$.

Peres, C. A. 1999. General guidelines for standardizing linetransect survey of tropical forest primates. Neotropical Primates 7(1):11-16.

Pinto Junior, O. B. \& Rossete, A. N. 2005. Caracterização morfométrica da bacia hidrográfica do ribeirão Cachoeira, MT-Brasil. Geoambiente on-line 4:38-53.

RADAMBRASIL. 1981. Levantamento dos Recursos Naturais. Ministério das Minas e Energia, 25, Folha SD - 22/ Goiás.

Ribeiro, J. F. \& Walter, B. M. T. 1998. Fitofisionomias do bioma Cerrado. In: SAno, S. M. \& Almeida, S. P. eds. Cerrado: ambiente e flora. Planaltina, EMBRAPA. p.89-166.

Stains, H. J. 1975 Distribution and Taxonomy of the Canidae. In: Fox, M. W. ed. The wild canids: their systematics behavioral ecology and evolution. New York, Van Nostrand Reinhold Company. p.3-26.

Thomas, L.; Buckland, S. T.; Burnham, K. P.; Anderson, D. R.; Laake, J. L.; Borchers, D. L. \& Strindberg, S. 2002. Distace sampling. In: El-ShaArawi, A. H. \& Piegorsch, W. W. eds. Encyclopedia of Environmetrics. Chichester, John Wiley \& Sons. p.544-552.

Thomas, L.; LaAke, J. L.; StrindBerg, S.; Marques, F. F. C.; Buckland, S. T.; Borchers, D. L.; Anderson, D. R.; Burnham, K. P.; Hedley, S. L.; Pollard, J. H.; Bishop, J. R. B. \& Marques, T. A 2005. Distance 5.0. Release " $x$ "1. Research Unit for Wildlife Population Assessment, University of St. Andrews, UK. Disponível em: <http://www.ruwpa.st-and.ac.uk/distance/>. Acesso em: 05.03.2006.

Tomás, W. M.; Mcshea, W.; Miranda, G. H. B.; Moreira, J. R.; Mourão, G. \& Borges, P. A. L. 2001. A survey of a pampas deer, Ozotoceros bezoarticus leucogaster (Artiodactyla, Cervidae), populations in the Pantanal wetland, Brazil, using the distance sampling technique. Animal Biodiversity and Conservation 24(1):101-106. 